\title{
Conceptual Model for the Evaluation of the Organizational Impact
}

\author{
Marco Tulio Cerón López, Verónica Flores, Manuel González Pérez
}

PhD Student in Strategic Planning and Management of Technology, México

Researcher, UTCV, México

Researcher SNI-1, Autonomous Popular University of the State of Puebla, Interdisciplinary Center of Postgraduate, Investigation and Consultancy

\begin{abstract}
The process of evaluating the organizational impact is a subject widely studied nationally and internationally. The reference to the organizational impact, it is necessary to focus on the positive or negative results of a change, modification or innovation applied to the organization and that directly affects a part or the entire organization. The Conceptual Model for the Evaluation of the Organizational Impact (CMEOI) to determine the degree of knowledge of the objective, goals or projects, information and communication, effectiveness of specific programs or projects, motivation, benefits, stimuli or compensation, labor climate and identification of potential. The present investigation proposes a conceptual model that will be useful to determine the degree of organizational impact, of an entity in the face of any change, modification or innovation, whether internal or external.
\end{abstract}

Keywords - Model concept, evaluation, organizational impact.

\section{INTRODUCTION}

The process of evaluation of the impact turns out to be a topic extensively national agreement and internationally. Starting for evaluating in the decade of 1960 the environmental impact, like a process of analysis and prevention on the changes that was suffering our ecosystem. Later, there begins talking each other the evaluation of the social impact like a wider concept, which was including the community. Of the same form, there are considered to be the effects, positives and negatives that turn out to be from the implementation of a certain program or project in a group a partner.

Speaking on the evaluation of the impact to the organizations, investigators agree in showing that it is the process of identifying, of obtaining and of providing useful information. The evaluation gives us the possibility of knowing and of comprising the effects applied to a project, program or strategy, as regards the goals or targets proposed, in order to serve as guide for the decision making.

\section{Organization}

An organization according to the definition that expresses the Rea Academy of the Spanish language is "association of persons regulated by a set of rules in the light of certain purposes" (Association of Academies of the Spanish Language, 2014).

An organization is defined like, an agreement debated between persons to expire with a specific intention, itself an individual of independent form they might not manage only. The main characteristics that the organizations are: they must have a definite intention (target or goals that the organization hopes to reach), it must be shaped by persons to realize the necessary work. Finally the organizations must be provided with a structure debated with the intention of which its members should have the possibility of carrying out its work (P. Robbins and Coulter, 2014).

Another reference of the term organization, the Dictionary of Economy her it defines like the different resources, activities or functions of the company that can be coordinated nor directed of the form that is convenient to the attainment of its targets without an organizational structure. The theoretical method or conceptual frame of reference for which he gets ready: what to do, how to do it, who has to do it and when it must be done. The organization is a consequence or result of the process of planning, by means of which from a few targets that are formed there are identified the functions, activities and works to be developed to reach them, and there get ready the resources, materials and human beings necessary to carry them out. Organizing is the deliberate attempt of adapting means to obtain ends (Papadakis, 2016).

We conclude the definition of an organization, like a social group composed by resources (materials, human beings and 
financiers), definite activities and an administration; that jointly form a systematical structure of relations of interaction, with the intention of producing goods and/or services to satisfy the needs for a community inside an environment. An organization, there are persons capable of communicating and they are ready to act jointly to achieve a common target, working by means of a set of rules and norms of behavior that the members adopt.

\section{Impact}

It is important to mention that the impact term for this investigation does not refer to the concept of blow of a bullet or of another object against anything or anybody, but to the effect that can produce an action, decision, news or event on an organization.

Initiating with the concrete impact definition, we have that the Royal Spanish Academy of the Spanish Language, it is the effect produced in the public opinion by an event, a disposition of the authority, news, a catastrophe, etc. (Association of Academies of the Spanish Language, 2014). We can specify the term I crash, as the expression of an effect to an action, was begun to use this concept in investigations and works on the environment. It is possible to mention, of allusive form, the definition of "environmental impact". Where one raises that it is said that there is environmental impact when an action or activity produces an alteration, favorable or unfavorable in the way or some of the components of the way. In the same way we can refer to the impact of a project on the environment, it is the difference between the current situation of the environment and future modified to the same one, as a result of the achievement of the project, that is to say, the positive or negative alteration in the quality of life of the human being, as resultant from a performance" (Bonilla and Esther, 2007).

\section{Organizational impact}

After contextualizing the concept of impact and organization, we them proceed to work with the intention of having the only term that serves to us as reference for it presents investigation. And thus we can define to the organizational impact like the change generated in the organization as a result of an innovation (Bonilla and Esther, 2007).

On the other hand we can consider to the competitions and high levels of organizationalcapital, as the training and the experience, they turn out to be closely related to the strategies of innovation of an organization. Generating like turned out a development of systems, routines and agile and flexible politics, taking like an impact as an organizational result(Suárez Núñez and Martín Méndez, 2008).

We can appreciate the change from the point of view of the transition, which consists of going on from one state to other and they can present themselves of three forms. The first ones are named evolutionary changes, which turn out to be slow and collaborate with natural processes of growth and organizationalconsolidation; the second ones are the revolutionary changes, which one recounts to those transformations that happen in a sudden, unexpected and abrupt way. And finally the systematical changes, which happen in a planned, constant but not too rapid way, being considered to be that in this one can be controlled or minimizing the impact to the organization (Osorio Jaramillo y Ravelo Contreras, 2011).

With base in the previous thing, we can determine that the organizational impact is the positive or negative result of a change, modification or innovation applied to the organization and that it affects in a direct way the same one orsomeone of its parts.

\section{Evaluation of the Impact}

Next, we will describe some elements to be thought inside the evaluation of the organizational impact and how it has been tackled in the different environments in which they have presented before themselves, what will give a panorama wider than it is the study topic.

Often it is difficult to go forward to changes in an organization or company if these are not based by the information, values, attitudes and conducts of its people (information), by what a commitment turns out to be important at the time of initiating them. In other words, to measurement into which the members of the organization integrate and are done copartner of the changes feels more protagonist of the processes and their not victim. If one thinks about how to add value and increase the competitiveness of the company, it is possible to reduce risks, to increase the productivity being based on finished and precise reports of the impact that has had the change inside the organization(Nava, Nava, \& Nava, 2011).

Within an organizational assessment, it is important to consider the communication that exists in the same. The communication within an organization is a process that takes place between members of the same. The communication within the organizations is in a dynamic activity, which is adjusted in accordance with the development of the organizations. The set of communication processes within organizations, are 
considered as systems. A system that is composed of a series of mutually reinforcing activities, that when integrated form a specific set of objectives. The communication between the members of an organization implies the creation, exchange (reception and sent), processing and storage of messages (Velázquez Valadez, 2012).

The human capital of an organization and it is in main asset for the generation of competitive advantages. To speak about the human capital is not to refer to professional training, knowledge, experiences, judgments, relations or interaction between workpeople of any hierarchic level. It is to refer to the attribute of the collections of individuals, the same that they include the structure, the formal and informal systems of planning and managerial control, as well as the culture and the informal relations between all what they interact in the same ambience; turning out to be an important concept to be evaluated when we tackle the organizational impact (Suárez Núñez and Martín Méndez, 2008).

Organizational Impact also is a part of the technological changes in the companies in the handling of the administrative processes, because there are a way of changing the way of doing the things, normally these processes happen of being complex and bureaucratic to a major automation and management. For side, the system inclusion of information, inside the organization, there are changes generators in the organizationalculture, going on from a traditional culture to an of integration culture. Existing one related to the administration of the knowledge, of the innovation and of the technology, what would not be possible inside the traditional administration (RiascosErazo and Aryans Cardona, 2016).

\section{RESEARCH PROPOSAL}

With base in the expressed for the different authors on the organizationalimpact, there is done the proposal of the Conceptual Model for the Evaluation of the Organizational Impact (CMEOI) that allows us to know aspects like:

- Grade of knowledge of the target, goals or projects.

- Information and communication.

- Efficacy of programs or specific projects.

- Motivation, benefits, stimuli or compensations.

- Work environment.

- Identification of potential.

For the consequent thing, the present work proposes a conceptual Model that is useful to determine the organizational impact to the one that sees an entity involved on having faced some change, modification or innovation, be already internal or external.

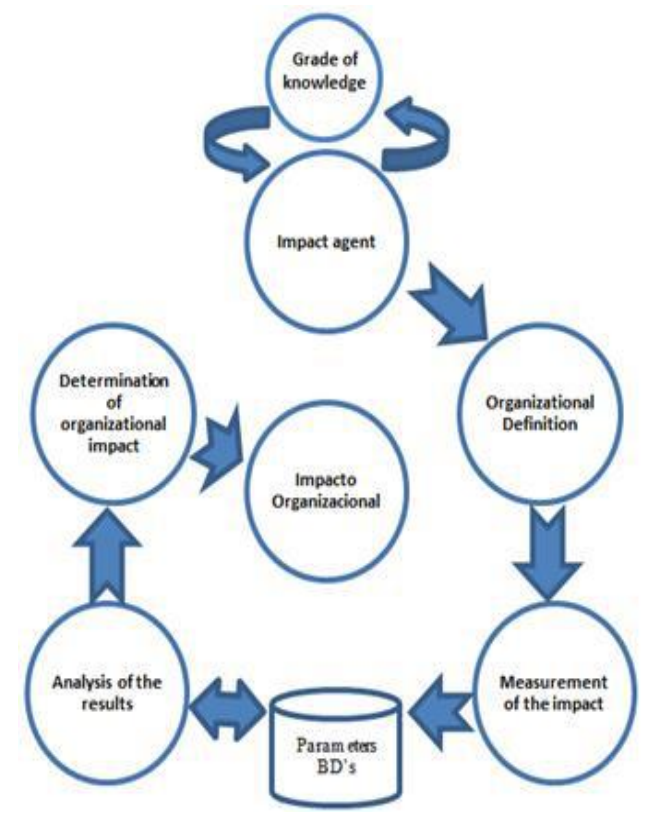

Conceptual Model for the Evaluation of the Organizational Impact (CMEOI)

\section{Impactagent}

The first step to be realized is to determine what will be the factor, change, innovation that he goes to analyze to determine the impact in the organization.

Of the agent, it is necessary to evaluate the grade of knowledge that the organization of the same one has and to analyze the perception that is had on the part of the members of the organization.

For example, if you pretend to analyze the implementation of a goal that search to concentrate effort to make the process of patenting to perform within the institution, it is necessary to determine aspects such as the following:

- Grade of knowledge of the agent of impact

- Understanding of the procedures to be realized

- Interest on the impact agent

With base in the results it is necessary to have in consideration the following options:

- If the grade of knowledge of the agent of impact is bigger than $51 \%$ it is measurable.

- Otherwise it will have to of recommending to him a sensitization process.

\section{Organizational Definition}

After knowing the agent of impact that will be studied, it is necessary to determine specifically the meeting of the Organization which have or will have involvement of a 
positive or negative way before the first point. Youmust define:

- Persons in charge

- Areas or involved departments

- Personnel to which it would impress the reagent

For the previous thing it is necessary:

- Determination of the size of the population.

- Determination of the sample, with base in the following formula:

$n=\frac{N \times Z_{a}{ }^{2} \times p \times q}{d^{2} \times(N-1)+Z_{a}{ }^{2} \times p \times q}$

Where, $\mathrm{N}=$ size of the population $\mathrm{Z}=$ confidence level, $\mathrm{P}=$ success probability, or awaited proportion $\mathrm{Q}=$ defeat probability $\mathrm{D}=$ precision (admissible maximum Error in proportion terms).

- To realize a probability sampling

\section{Measurement of the impact}

To carry out the measurement of the impact it is necessary to design a methodology that it allows us to investigate in a world of possibilities and probabilities. For example the quantum methodology that is based on the beginning of the suspense, same that he studies the options multiplicity and calculates probabilities.

It is necessary to create an instrument of compilation of information with base in the indicators to decide in the point 1 of the Conceptual Model for the Evaluation of the Organizational Impact(MCEOI).

It can be an opinion poll, applied to the personnel defined in the stage 2 of the CMEOI, which allows us to obtain the necessary information.

\section{Analysis of theresults}

The analysis of the information obtained in the previous point, it owes it will realize by means of the application of the statistical model of distribution of continuous probability how it is the Pearson distribution, called also ji squared (o) or chi square (a) $\left(\chi^{2}\right)$.

The void hypothesis of the test Chi-Square postulates a distribution of probability completely specified like the mathematical model of the population who has generated the sample.

After having the result, it is necessary to do a comparison with the Database of Parameters, same that it must contain the results of at least 3 organizations and realize analysis parametric for the comparison of averages (ANOVA), which will help us to determination of parameters of analysis.

\section{Determination of organizationalimpact}

Finally, the determination of the organizational impact is in function of the official of impact on the definition of the organization that was studied. Resulting in a contract between the data, we will define the degree of organizational impact.

\section{CONCLUSION}

The measurement of the organizational impact is an integral process, where we glimpse the degree of knowledge of the members of the organization on a concept in specific that $i$ have known as agent of impact, define the competition of the area and of the members of the organization that are affected by this agent, to make an assessment of the organizational impact.

The Conceptual Model for the Evaluation of the Organizational Impact that allows us to define a methodology that allows to measure the organizational impact, resting of an instrument of compilation of information that allows to do an analysis of the information and this way, finally to measure in a precise way the positive or negative impact of the agent of impact.

The proposal of the Model MCEOI, it is presented of conceptual form with the target to fix the base of a practical application and demonstrate turned out concrete on the impact that an organization suffers and sayings turned out will be presented one future publications.

\section{REFERENCES}

[1] Association of Academies of the Spanish language. (2014). Dictionary of the Spanish language. Madrid: Espasa.

[2] Bonilla, L., \&; Esther, B. (2007). Impact, social impact and impact assessment. ACIMED, 0-0.

[3] Nava, M., Nava, R., \&; Nava, M. (2011). Organizational impact of innovation and information technologies. Formation managerial, 88-107.

[4] Osorio Jaramillo, L., \&; Ravelo Contreras, E. (2011). Perceived Impact of organizational change in workers of a university institution in Bogota: an approximation hermeneutics. Psychologia: progress of the discipline, 80-94.

[5] P. Robbins, S., \&; Coulter, M. (2014). Administration. Mexico: Addison-Wesley. 
[6] Papadakis, A. (2016). The Gran Enciclopedia of economy. Cyprus: Theodakis Publishing Ltd.

[7] Riascos Erazo, S., \&; Arias Cardona, V. (2016). Analysis of the organizational impact of the process of implementation of the Information Systems ERP. Framing, 284-302.

[8] Suarez Núñez, T. F., \&; Martin Mendez, M. (2008). The impact of human capital and organizational capital on SME strategies. Notebooks of Administration, 229248.

[9] Suarez Núñez, T. F., \&; Martin Mendez, M. (2008). Impact of the capital rights and organizational strategies for SMES. Notebooks of Administration, 229-248.

[10] Velazquez Valadez, G. (2012). Impact of the organizational communication, in the competitiveness and the linking of mexican companies with the university. Recherches in Sciences de Gestion, 89-119. 